\title{
Laparoscopic Cholecystectomy for Large/Giant Gallstones: Case Report and Brief Review of Literature
}

\author{
Anup Shrestha', Shachee Bhattarai', Shreya Shrestha', Manoj Chand', Abhishek Bhattarai' \\ 'Department of Surgery, Chitwan Medical College Teaching Hospital, Bharatpur, Nepal
}

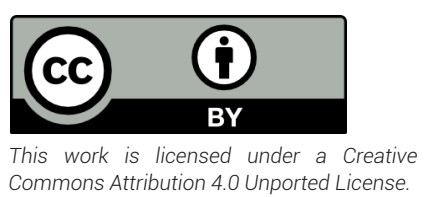

\begin{abstract}
Gallstones disease are the most common biliary pathology. Its prevalence in Nepal is found to be $4.87 \%$. Giant/large gallstones have a higher risk of complications and presents technical difficulties during laparoscopic cholecystectomy. Open cholecystectomy is preferred in most of the cases with giant gallstones. With the availability of experience laparoscopic surgeon and modern laparoscopic equipment, laparoscopic cholecystectomy is also feasible in large/giant gallstones. We report 2 cases, one large gallstone in 51 years old female and one giant gallstone in 39 years old female each of which were successfully managed laparoscopically with uneventful post-operative period.
\end{abstract}

\section{KEY WORDS}

Large; Giant; gallstone; Cholecystectomy; Laparoscopic

\section{BACKGROUND}

Gallstone disease(GSD) are the most common biliary pathology. 'The estimated effect of $10-15 \%$ of the population in Western societies. ${ }^{1}$ It is also a significant cause of morbidity in Nepal. Its prevalence is found to be $4.87 \%$. The ratio between male and female was 1:2.2 Laparoscopic Cholecystectomy (LC) is the gold standard for GSD and it is one of the most common operation performed by surgeons. ${ }^{2}$

The size of a gallstone is important, as giant/large gallstones have a higher risk of complications and higher technical difficulties during laparoscopic cholecystectomy. Gallstones $>3 \mathrm{~cm}$ are called large gallstones and they carry a higher risk for gallbladder cancer. Gallstones> $5 \mathrm{~cm}$ are called giant gallstones and they are very rare with only very few cases reported in the literature. ${ }^{4}$ In this case report which is first from Nepal, we report 2 cases of one large and one giant gallstone each which were successfully done laparoscopically and also review the literature.

\section{CASE 1:}

A 51 years, female presented to the surgical outpatient department at our institution with history of intermittent right upper quadrant colicky pain for 5 months which was related to fatty food, not radiating and not associated with fever or jaundice.

On examination, vital signs and abdominal examination were unremarkable. The laboratory parameters were normal. Ultrasound of the abdomen showed gallbladder with multiple gallstones, the largest measuring approximately 4 cms with no features of acute cholecystitis.

Diagnosis of symptomatic cholelithiasis was established and was planned for elective LC. During operation, after pneumoperitoneum was created, there were dense adhesions found between the greater omentum and gallbladder (body and fundus) which were released with the help of harmonic scalpel. The gallbladder was distended with a short cystic duct. The gallbladder wall was thick and the gallstone occupied almost the entire Hartmann's pouch which caused grasping of the gallbladder by non-traumatic forceps difficult.

After achievement of the critical view of safety the clipping and division of the cystic artery and duct was done. A drain was placed at subhepatic region. Then the gallbladder was dissected from the cystic plate, put in an endobag and removed out after extension of the infra umbilical incision. On cut section we found multiple gallstones with one large gallstone measuring $4 \times 3.3 \times 3 \mathrm{~cm}$ and weighted $23.2 \mathrm{gm}$ (Figure 1).

\section{*Corresponding Author}

Anup Shrestha, Department of Surgery, Chitwan Medical College and Teaching Hospital, Chitwan, Nepal, Email: anup.10281028@gmail.com, Mobile number. +9779856015288 


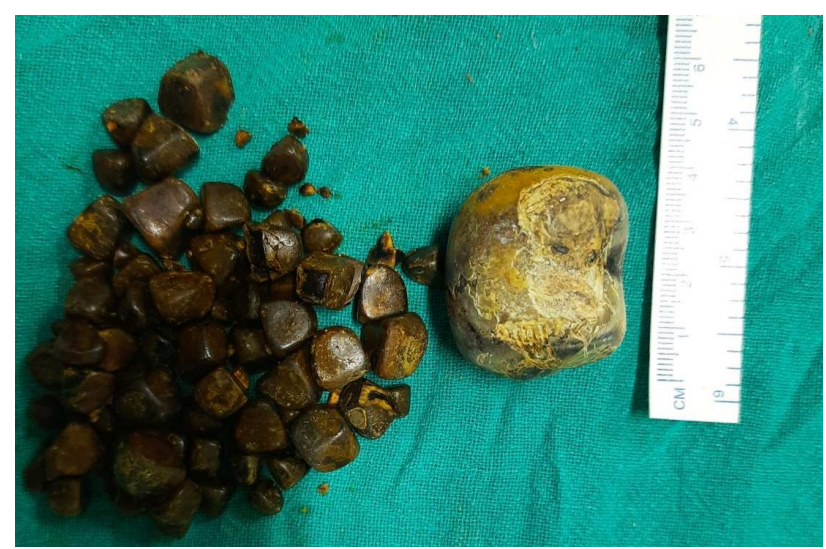

Figure 1: Multiple gallstones with a large stone with measuring scale

Post-operative course was uneventful and the drain was removed on second post-operative day. The patient was discharged on third post-operative day. The histopathological report showed chronic follicular cholecystitis and no evidence of malignancy.

\section{CASE 2}

A 39 years, female presented to the surgical outpatient department at our institution with a 3 months history of intermittent right upper quadrant colicky pain related to fatty foods, not radiating, and not associated with fever or jaundice. There was a past medical history of hypertension for which she was under medication. There was no history of previous abdominal surgery

On examination, her vital signs and abdominal examination were unremarkable. Investigations showed laboratory parameters within normal limits. Ultrasound of the abdomen showed a gallbladder with a single large gallstone (approximately $4.7 \mathrm{~cm}$ ) and no features of acute calculus cholecystitis.

The patient was diagnosed with symptomatic cholelithiasis and was admitted for elective LC under general anesthesia. Intraoperatively, there were some adhesions between the gallbladder and duodenum which were released. The gallbladder wall was thick and the gallstone occupied almost all of Hartmann's pouch which rendered grasping the gallbladder difficult.

After achieving critical view of safety and clipping the cystic artery and duct was done and the gallbladder was dissected from the cystic plate. It put in an endobag and removed out after extension of the infraumbilical incision. On cut section, we found a single giant gallstone measuring $5.2 \times 3 \times 2.8 \mathrm{~cm}$ and weighted $24.7 \mathrm{gm}$ (Figure 2).

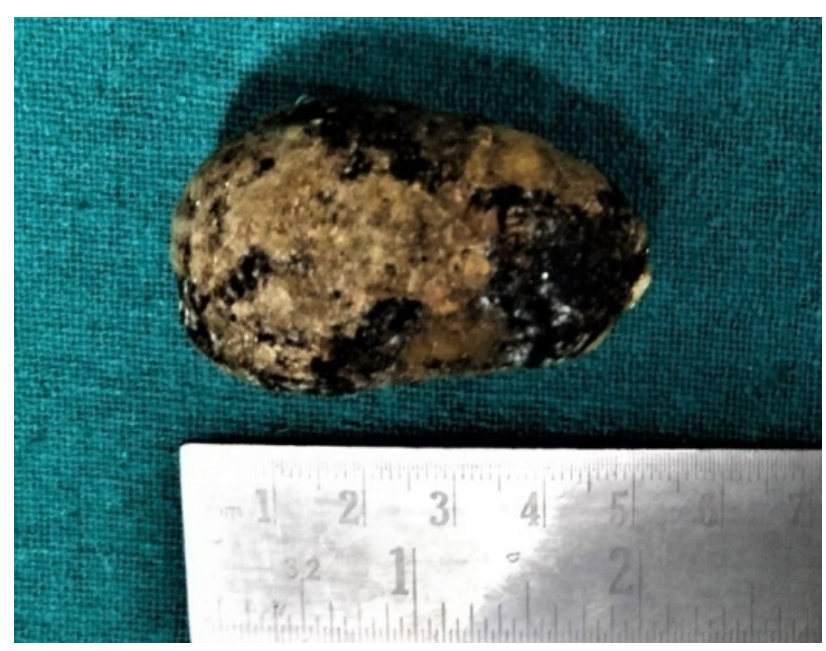

Figure 2: Single giant stone extracted with measuring scale

The post-operative course was uneventful and the patient was discharged on second post-operative day. The histopathological report showed chronic cholecystitis with pyloric metaplasia and no evidence of malignancy.

\section{DISCUSSION}

The case report of largest gallstone removed by LC was reported by Singh et al. which was $12.8 \mathrm{~cm}$ in diameter. ${ }^{5}$ Few other reports have been reported for giant gallstones. ${ }^{6,7}$ We report two cases of patients with large/giant gallstones. In the first case, gallstone measured $4 \times 3.3 \times 3 \mathrm{~cm}$ and weighted $23.2 \mathrm{gm}$ and in the second case, gallstone measured $5.2 \times 3 \times 2.8 \mathrm{~cm}$ and weighted $24.7 \mathrm{gm}$. To the best of our knowledge, this is the only case report of large/giant gallstones reported till now in the literature in Nepal. Both cases were laparoscopically managed, and their postoperative courses were uneventful with no complications. Both patients were followed up after two weeks, where they had no active complaints.

As for demographics, GSDs are more common in women, especially during their fertile years, probably due to increased estrogen levels which may increase cholesterol in the bile and decreased gallbladder movement, resulting in gallstone formation. ${ }^{8}$ Our case reports are in agreement, as both the cases are female. In terms of age, the frequency of gallstones increases with age, escalating after 40 years of age to become 4-10 times more likely. ${ }^{8}$ Our first patient is 51 years old whereas second patient is 39 years old, slightly younger than other reports. North Americans have the highest GSDs rates, South Americans also have high prevalence, intermediate prevalence rates occur in Asians and Black Americans, and sub- Saharan Black Africans have the lowest frequencies. ${ }^{8}$ GSDs is one of the common surgical problems in Nepal and its prevalence is $4.87 \%$. Highest prevalence found to be in Morang (6.45) and lowest in Achham (2.44). ${ }^{2}$ 
As for presentation, $60-80 \%$ of gallstones are asymptomatic frequently found during routine abdominal ultrasonography. ${ }^{9}$ Symptomatic gallstones may present as biliary pain, cholecystitis or biliary obstruction depending on location. ${ }^{10}$ In agreement, our cases presented as biliary pain. Gallstones can also present as gallstone ileus especially in large gallstones causing bowel obstruction. ${ }^{11}$

Ultrasonography is the method most often used to detect cholelithiasis and cholecyctitis (90-95\% specificity and sensitivity), can detect and accurately assess stone size as small as $2 \mathrm{~mm}$, show thickening of the gallbladder wall, and should be routine.12 Ultrasonography has advantage e.g. lack of ionizing radiation, noninvasiveness, option of performing a bedside examination, relatively low cost and ability to evaluate adjacent organs. ${ }^{12}$ For our two patients, abdominal ultrasonography showed the size of the giant gallstones, with measurements close to the actual size found after surgery. Such accurate pre-operative assessment of a giant gallstone alerts the surgeon to any potential difficulty of the procedure and the possibility of conversion to open cholecystectomy. This allows the surgeon to be prepared and to explain the potential rates of complications to the patient. ${ }^{6}$ We were prepared in terms of surgical instruments and settings for a possible conversion to open at any point during the surgery.

Most gallstone patients remains asymptomatic. ${ }^{10}$ Asymptomatic gallstones $>3 \mathrm{~cm}$ are at higher risk to develop gallbladder cancer and hence LC is warranted. ${ }^{13}$ For symptomatic gallstones, LC has become the management of choice. For giant gallstones, some authors believe open cholecystectomy is the choice, given the technical difficulties related to the stone's large size that could be confronted during the laparoscopic approach. ${ }^{14}$ However, we believe that even with giant gallstones, LC performed by an experienced laparoscopic surgeon is still the best initial approach, unless technical difficulties and inability to expose the anatomy warrants conversion to open cholecystectomy. ${ }^{6}$ We used laparoscopic approach for our patients without need for conversion, there were no intra- or post-operative complications, and recovery was uneventful.

Giant gallstones could cause result in severe inflammation, adhesions and thickening of the gallbladder wall, where adhesions is an important reason for conversion of laparoscopic to open cholecystectomy. In addition, giant gallstones make it difficult to grasp the gallbladder with laparoscopic instruments and expose the anatomy of Calot's triangle..$^{15}$ We faced the same difficulties in our two cases, where the main challenge was to release the adhesions between the gallbladder and surrounding structures, and to hold the thickened and inflamed gallbladder wall by the laparoscopic grasper prior to starting dissection.
Another consideration is the site and manner of retrieval of the gallbladder out of the abdomen after cholecystectomy. We retrieved the gallbladder through the umbilical approach in both cases, after infraumbilical extension of wound. In terms of manner of retrieval, for our cases, the gallbladder was put in an endobag before taking it out of the abdomen to prevent spillage of bile or wound infection, in line with a recent meta-analysis that found that the wound infection rate was less in patients who underwent retrieval of gallbladder using a bag vs without (4.2\% vs $\% 5.9 \%)^{17}$

\section{CONCLUSION}

Large/giant gallstones are associated with high risk of complications and LC is warranted in symptomatic and asymptomatic patients. Even for large/giant gallstones, LC appears to be the treatment of choice over open cholecystectomy and should be performed by experienced laparoscopic surgeons, taking into consideration the possibility of conversion to open in case of inability to expose the anatomy and any intraoperative technical difficulties.

\section{ABBREVIATIONS}

LC

Laparoscopic Cholecystectomy

GSD Gallstone Disease

CCB Calcium Channel Blocker

ARBs Angiotensin Receptor Blockers

\section{Consent for publication}

Written informed consent was obtained from the patient or patient's legal guardians for publication of this case report and any accompanying images. A copy of the written consent is available for review by the Editor-in-Chief of this journal.

\section{Competing interests}

The authors declare that they have no competing interest to disclose.

\section{Funding details}

No funding was required for the study.

\section{Author's Contributions}

Anup Shrestha and Shachee Bhattarai did the main manuscript writing and literature review. Shreya Shrestha did the final manuscript editing and design. Manoj Chand collected pictures, and did language edition and manuscript review, Abhishek Bhattarai did the study concept, literature review, and was the main operative surgeon in both the cases. All the authors approved the final manuscript. 


\section{REFERENCES}

1. Neupane RP, Shrestha TM, Raut S, Aacharya RP. Risk Factors for Gall Stone Diseases in Patients Presenting to General Practice Out Patient Department in a Tertiary Care Center in Nepal. J Inst Med Nepal. 2019;41(2):26-29. doi:10.3126/jiom. v41i2.26545

2. Mukund Raj Panthee, Yagya Raj Pathak APA, Chakradhar Mishra RKJ. Prevalence of Gall Stone Disease in Nepal : Natl Acad Med Sci -NAMS PMJN. 2007;7:45-50.

3. Conlon K. The gall bladder and bile ducts. In: Williams NS, Bulstrode CJK OP, ed. Bailey and Love's Short Practice of Surgery. 26th ed. FL: CRC; 2013:1097-1117.

4. Al Zoubi M, El Ansari W, Al Moudaris AA, Abdelaal A. Largest case series of giant gallstones ever reported, and review of the literature. Int J Surg Case Rep. 2020;72:454-459. doi:10.1016/j. ijscr.2020.06.001

5. Singh $Y$, Mohammed S, Hosein A, Ramoutar K, Naraynsingh V. A Giant Gallstone: The Largest Gallstone Removed Laparoscopically in the World. Cureus. 2020;12(5):1-7. doi:10.7759/cureus.7933

6. Xu X, Hong T, Zheng C. Giant gallstone performed by emergency laparoscopic cholecystectomy. Int J Surg Case Rep. 2013;4(12):1163-1164. doi:10.1016/j.ijscr.2013.10.002

7. Igwe PO, Diri ON. CASE REPORT-OPEN ACCESS Laparoscopic cholecystectomy for giant gall stone: Report of two cases. Int J Surg Case Rep. 2020;67:207-210. doi:10.1016/j. ijscr.2020.01.055

8. Stinton LM, Shaffer EA. Epidemiology of gallbladder disease: Cholelithiasis and cancer. Gut Liver. 2012;6(2):172-187. doi:10.5009/gnl.2012.6.2.172

9. Schmidt M, Hausken T, Glambek I, Schleer C, Eide GE, Søndenaa K. A 24-year controlled follow-up of patients with silent gallstones showed no long-term risk of symptoms or adverse events leading to cholecystectomy. Scand J Gastroenterol. 2011;46(7-8):949-954. doi:10.3109/00365521.2011.571710

10. Attili AF, de Santis A, Capri R, Repice AM, Maselli S, Group G. The natural history of gallstones: The GREPCO experience. Hepatology. 1995;21(3):656-660. doi:10.1002/hep.1840210309

11. Freeman MH, Mullen MG, Friel CM. The Progression of Cholelithiasis to Gallstone Ileus: Do Large Gallstones Warrant Surgery? J Gastrointest Surg. 2016;20(6):1278-1280. doi:10.1007/s11605-016-3096-0

12. Bortoff GA, Chen MYM, Ott DJ, Wolfman NT, Routh WD. Gallbladder stones: Imaging and intervention. Radiographics. 2000;20(3):751-766. doi:10.1148/ radiographics.20.3.g00ma16751

13. Csendes A, Becerra M, Rojas J, Medina E. Number and Size of Stones in Patients with Asymptomatic and Symptomatic Gallstones and Gallbladder Carcinoma: A Prospective Study of 592 Cases. J Gastrointest Surg. 2000;4(5):481-485. doi:10.1016/S1091-255X(00)80090-6
14. Of J, Reports C. 17 Journal of Case Reports, Vol. 4, No. 1, JanJune 2014. 2014;4(1):17-19.

15. Kama NA, Doganay M, Dolapci ME, Reis M, Atli M KM. Risk factors resulting in conversion of laparoscopic cholecystectomy to open surgery. Risk factors resulting Convers Laparosc cholecystectomy to open Surg. 2001;15(9):965-968.

16. Hajibandeh S, Hajibandeh S, Clark MC, et al. Retrieval of Gallbladder Via Umbilical Versus Epigastric Port Site during Laparoscopic Cholecystectomy: A Systematic Review and Meta-Analysis. Surg Laparosc Endosc Percutaneous Tech. 2019;29(5):321-327. doi:10.1097/SLE.0000000000000662

17. Sandstrom P, Bjornsson B. Bile spillage should be avoided in elective cholecystectomy. Hepatobiliary Surg Nutr. 2019;8(6):64042-64642. doi:10.21037/hbsn.2019.07.14 